\title{
Preparation of Fluorescent Molecularly Imprinted Polymer Micropowder for Odorant Visualization
}

\author{
Kazuya Iwata, ${ }^{1}$ Seichi Yamashita, ${ }^{2}$ Hiro-Taka Yoshioka, ${ }^{2}$ \\ Chuanjun Liu, ${ }^{3 *}$ and Kenshi Hayashi ${ }^{1,2,3}$ \\ ${ }^{1}$ Graduate School of Systems Life Sciences, Kyushu University, \\ 744, Motooka, Nishi-ku, Fukuoka, 819-0395 Japan \\ ${ }^{2}$ Graduate School of Information Science and Electrical Engineering, Kyushu University, \\ 744, Motooka, Nishi-ku, Fukuoka, 819-0395 Japan \\ ${ }^{3}$ Research and Development Center for Taste and Odor Sensing, Kyushu University, \\ 744, Motooka, Nishi-ku, Fukuoka, 819-0395 Japan
}

(Received September 18, 2015; accepted December 25, 2015)

Keywords: molecularly imprinted polymer, fluorescent micropowder, odorant visualization, selectivity, reversibility

Odorant visualization based on fluorescence imaging has been developed as a novel technique to obtain spatiotemporal information on odors in an environment. In order to increase the selectivity and reversibility of sensing films, fluorescent-dyed micropowders with molecular imprinting ability were proposed for the odorant visualization. The micropowders were prepared by bulk polymerization in the presence of template and fluorescence probe molecules. The fluorescence emission character and the molecular imprinting effect of the prepared micropowders were investigated by fluorescence microscope observations and gas chromatography-mass spectrometry (GC/MS) measurements, respectively. The dyed molecular imprinted polymer (MIP) micropowders were coated on glass substrates and used as the sensing film for the selective visualization of odorant flows. The molecular imprinting effect was confirmed by the change in the ratio of the fluorescence of the MIP film to the non-imprinted polymer (NIP) film. In addition, a higher ratio was demonstrated by the MIP film on the target odorant than the non-target odorant, which verified the selective visualization of the prepared micropowder. The film also showed advantage in reversible response on various odorants; therefore the fluorescent MIP micropowder can be used repeatedly in the real time odorant visualizaiton.

\section{Introduction}

As information for one of the five senses (sight, sound, touch, smell, and taste), olfactory information plays an important role in the life of humans and other creatures. The olfactory information obtained by our noses includes not only the quality and intensity of odorants from different sources, but also their spatial/temporal distribution in the environment. The development of sensors with abilities similar to those of creatures will have a number of potential applications. Such sensors can be loaded on mobile robots to imitate the odor-locating behavior of creatures and thus could be applied to odor source localization, hazardous chemical detection, and

${ }^{\bar{*}}$ Corresponding author: e-mail: liu@o.ed.kyushu-u.ac.jp 
rescue. ${ }^{(1-7)}$ Although great achievements have been made in the development of gas/odor sensors, most research focuses on the detection of odor quality and intensity, while the spatial/temporal distribution information has been ignored. Moreover, sensing technologies based on conventional sensors, such as metal oxide semiconductors (MOSs), composite polymer chemiresistors, and quartz crystal microbalances (QCMs), are not suitable for the detection of spatiotemporal information of odorants, owing to either the limited response/recovery time of sensor elements or the poor spatial resolution of the sensor arrays. ${ }^{(1,5,8,9)}$

In our previous work, a fluorescence imaging sensor system was successfully developed to obtain spatiotemporal information on odorants in the environment. ${ }^{(10)}$ The system consists of an excitation light source, an agarose gel-sensing film containing a specific fluorescent probe, and a highly sensitive, cooled charge-coupled device (CCD) camera. The change of fluorescence intensity (such as quenching and enhancing) of the sensing film caused by interactions between the fluorescent probe and odorants can be recorded by the CCD camera and then visualized via imaging analysis. The sensor can be used to visualize not only the shape and size of odorant sources remaining on the substrate, but also the time-averaged spatial distribution of odorant plumes in a real environment. ${ }^{(11)}$ In addition, odorants are invisible to our eyes, and we can only perceive them by our olfactory system. However, with the aid of odorant visualization, the olfactory perception can be conveyed into visual perception, which provides an intuitive approach for us to perceive odors with our eyes instead of our noses. As a novel approach to obtain odor information that conventional sensors cannot acquire, we have explored many new applications for fluorescence imaging sensors in fields related to odor sensing. ${ }^{(12-14)}$

The key component of the fluorescence imaging device is the sensing film of agarose gel containing fluorescent probes. The spatially diffused odorants are absorbed by the gel and react with the probe, which results in changes of fluorescence intensity of the probes. Although the gel film shows great advantages in recording odorant shape, size, and distribution, its deficiencies are that the response is irreversible and the film cannot be reused. The gel film contains $95 \%$ water as its main component, and thus it is unwieldy to use owing to the poor mechanical strength of the gel materials. In addition, the fluorescent film based on single probes shows a lack of selectivity to odorants or their mixtures. In order to address these problems, new odorant visualization based on fluorescent-dyed polymer micropowders with molecularly imprinted character was tried. The MIP technique is introduced to increase the selectivity of the sensing film. The fluorescent-dyed micropowders are expected to increase the reversibility and repeatability of the fluorescence response to odorants. MIP micropowders were prepared using bulk polymerization in the presence of both templates and fluorescent dyes. The selective adsorption character and the visualization effect of the prepared fluorescent MIP micropowders were evaluated in this study.

\section{Experiment}

\subsection{Chemcials}

Methacrylic acid (MAA), trimethylolpropane trimethacrylate (TRIM), azodiisobutyronitrile (AIBN), quinine sulfate (QS), acetonitrile, hexanoic acid (HA), propionic acid (PA), and hydrochloric acid $(\mathrm{HCl})$ were used as analytical reagents and purchased from Wako Pure Chemical Induxtries, Ltd. (Japan). 


\subsection{Preparation of fluorescent MIP micropowder}

The fluorescent micropowders were prepared using bulk polymerization. QS (93.36 mg, 0.24 mmol) was first dissolved in a mixture of $4.5 \mathrm{ml}$ acetonitrile and $2 \mathrm{ml} \mathrm{HCl} /$ ethanol solution (1 M). Then, $0.38 \mathrm{ml}(4.5 \mathrm{mmol})$ MAA, $4.31 \mathrm{ml}(13.5 \mathrm{mmol})$ TRIM, $0.18 \mathrm{ml}$ (1.5 mmol) HA as the template, and $24.63 \mathrm{mg}(0.15 \mathrm{mmol}) \mathrm{AIBN}$ were added into the solution. The mixture was degassed by $\mathrm{N}_{2}$ purge for 10 min to remove oxygen. The solution was sealed and reacted at 90 ${ }^{\circ} \mathrm{C}$ for $8 \mathrm{~h}$. The resultant polymers were ground and screened with $100 \mu \mathrm{m}$ mesh. The powders were washed with ethanol 3 times and then heated in vacuum at $70{ }^{\circ} \mathrm{C}$ for $24 \mathrm{~h}$. This process was confirmed to be effective to remove the template molecules. The non-imprinted polymer (NIP) was similarly prepared in the absence of the template molecules.

\subsection{Odorant absorption evaluation by solid phase microextraction (SPME)-gas chromatography-mass spectrometry (GC/MS)}

The absorption character of the fluorescent MIP micropowders was evaluated by GC/ MS (QP2010SE, Shimadzu, Japan) with SPME probe. A divinylbenzene/carboxen on polydimethsiloxane (CAR/DVB on PDMS) 50/30 $\mu \mathrm{m}$ fiber (Supelco, Bellefonte, PA, USA) was used for SPME auto-sampling. The column used was a $60 \mathrm{~m}, 0.32 \mathrm{~mm}$ i.d., $0.30 \mu \mathrm{m}$ film thickness DB-WAX, and the carrier gas was helium. One piece of kimwipe soaked with $30 \mathrm{ml}$ liquid odorant was placed in a desiccator for $4 \mathrm{~h}$ to obtain saturated vapor. Then, a micropowder sample $(1 \mathrm{~g})$ was placed in the desiccator for $1 \mathrm{~h}$ to absorb the odorant. After the absorption, the micropowder was transferred to a vial for the SPME measurement. The extraction conditions of SPME were $90{ }^{\circ} \mathrm{C}$ and $15 \mathrm{~min}$. The SPME probe was desorbed in the injection port of the GC using an inlet temperature of $240{ }^{\circ} \mathrm{C}$ and a time of $30 \mathrm{~min}$. A $53 \mathrm{~min}$ program began with an initial oven temperature of $40{ }^{\circ} \mathrm{C}$ for $5 \mathrm{~min}$, followed by a ramp of $5{ }^{\circ} \mathrm{C} / \mathrm{min}$ to $230^{\circ} \mathrm{C}$, and ending with a 10 min hold. The quadrupole mass analyzer was operated in electron ionization mode, and the scan range was $\mathrm{m} / \mathrm{z} 35-550$.

\subsection{Odorant visualization}

The fluorescence imaging system shown in Fig. 1 was the same as that reported in our previous work. ${ }^{(11-13)}$ The system consists mainly of three parts: the excitation light source, the fluorescence

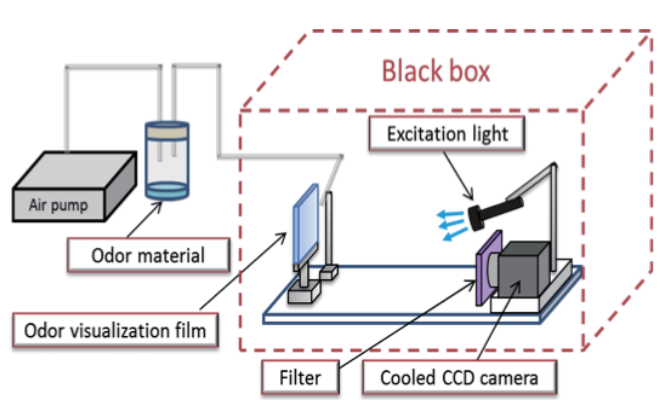

(a)

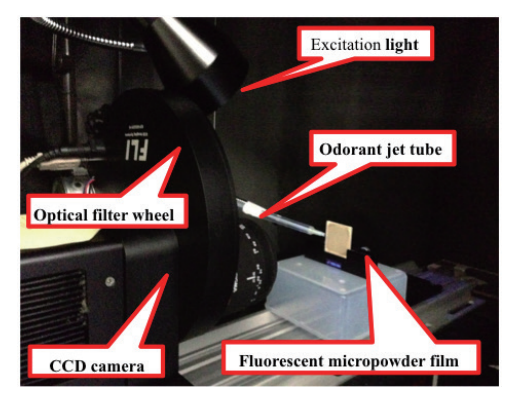

(b)

Fig. 1. (Color online) (a) Schematic diagram of fluorescence imaging sensor system and (b) picture of the photophgraphic system in the black box during the sensing process. 
sensing film, and the CCD camera. In this study, the micropowder sensing film was prepared by spin coating $(1000 \mathrm{rpm}, 3 \mathrm{~s})$ a $0.5 \mathrm{ml}$ suspension of the fluorescent micropowder $(0.5 \mathrm{~g} / \mathrm{ml}$ in ethanol) on a $3 \times 3 \mathrm{~cm}^{2}$ quartz glass plate. The plate was set on a stage between the CCD camera and a black screen at a suitable distance and height. For the visualization of odorant flow, odorless air was ejected to the surface of the film first, and then the flow was switched to odorant samples. The fluorescence images of the film under the excitation light were recorded successively by the CCD camera. The excitation light with a wavelength ranging from 300-400 nm was provided using an attached mirror module (LAX-UVA). The bandpass filter used on the camera side had a wavelength of $\sim 430( \pm 5) \mathrm{nm}$. The images were processed by the image calculator of ImageJ software using a subtract operation to visualize the odorant flow. The change of mean gray value (MGV) vs. time was extracted from the subtraction images to evaluate the time response character of the film to odorant flow.

\section{Results and Discussion}

QS was used in this study to dye the polymer in situ during the polymerization. QS can display two $\mathrm{pH}$-dependent emission peaks ( $\sim 380$ and $\sim 450 \mathrm{~nm}$ ) corresponding to its dicationic and monocationic structures. We used this character in our previous work to visually detect organic acid vapors. ${ }^{(13)}$ The fluorescence microscopy image shown in Fig. 2 confirms that the polymer micropowder was successfully dyed by QS. The observed fluorescence at a range of 420-460 $\mathrm{nm}$ agrees with the emission wavelength of QS with dicationic structure. The polymer matrix is poly(methacrylic acid) (PAA) crosslinked with TRIM. The carboxyl groups of the PAA matrix might enhance the protonation degree of QS, which contributed to the strong fluorescence emission of QS at long wavelengths $(\sim 450 \mathrm{~nm})$. The microscopic image also demonstrated that the size of the prepared micropowder was about 5-10 $\mu \mathrm{m}$. Although the size of micropowders was not regular, the inset in Fig. 2 demonstrates that they could be coated on the quartz glass substrate to form a homogeneous film, which showed fluorescence under the excitation light.

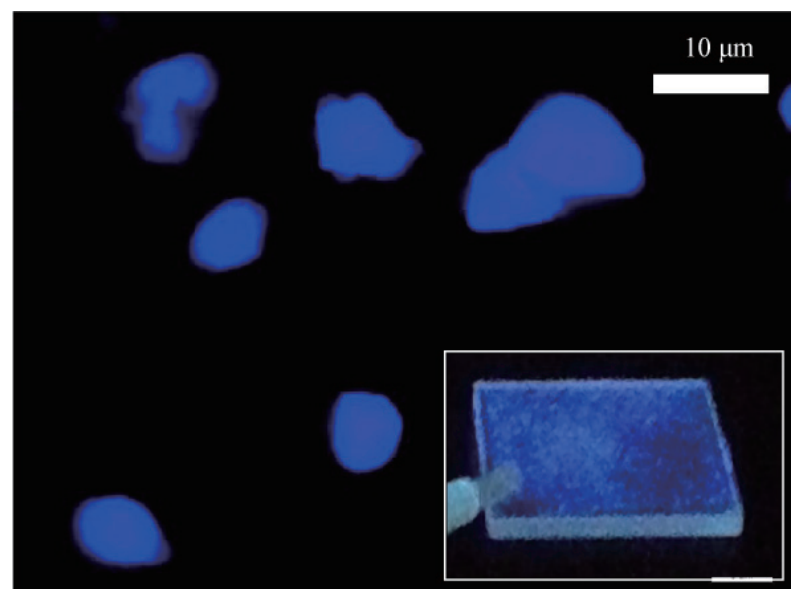

Fig. 2. (Color online) Fluorescence microscope image of the prepared micropowders $\left(\lambda_{\mathrm{ex}}=340-390 \mathrm{~nm}, \lambda_{\mathrm{em}}\right.$ $=420-460 \mathrm{~nm})$. Inset is the fluorescence image of the micropowder film coated on a quartz glass plate under excitation light. 
In order to confirm the visualization of the fluorescent micropowder, the NIP micropowder was used in the visualization of different odorants: HA and PA. As shown in Fig. 3(a), both odorant flows were successfully visualized. The fluorescence enhancement was shown for the organic acid vapors, which is same as that observed in QS-containing agarose gel film. Although the amount of fluorescence change was less than that observed in gel film, it is enough to visualize the odorant flows. The mean gray values (MGVs) of the sequence images were extracted from the sequence images to demonstrate the time response character of the micropowder film. Figure 3(b) illustrates the fluorescence change of the film with PA. The MGVs were extracted from the circled areas and plotted vs time. It can be seen that the film responded to odorant flow quickly and recovered to the baseline completely. In addition, the film can be used repeatedly without obvious fluorescence decay. Therefore, the film can be reused for odorant visualization.

The QS-dyed micropowder showed a fluorescence response to HA and PA, which makes it difficult to discriminate the two organic acid vapors. In order to increase the selectivity of the fluorescence response, the micropowder was prepared using MIP technologies. MIP has been widely used to prepare materials with selective molecule recognition ability. The process usually involves polymerization in the presence of a template molecule that is extracted afterwards, thus leaving complementary cavities behind. The polymers have affinity for the original or pseudo molecules and have been used in applications such as chemical separation, catalysis, and sensors. The affinity of the MIP materials can be evaluated by determining the imprinting factor (IF), which is defined by the absorption ratio of MIP to NIP. A strong molecular imprinting effect can be expected when there exist strong interactions between the functional monomers and template

Propionic acid

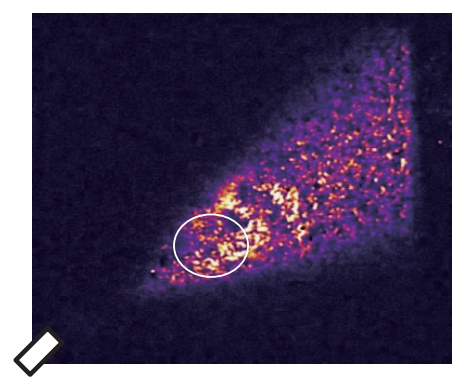

Hexanoic acid

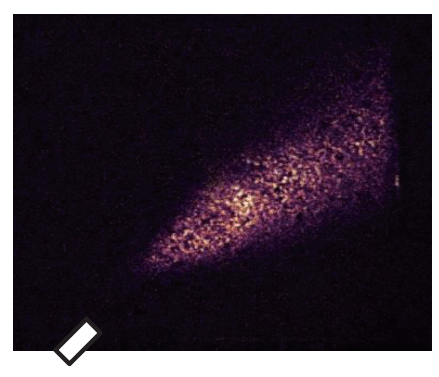

(a)

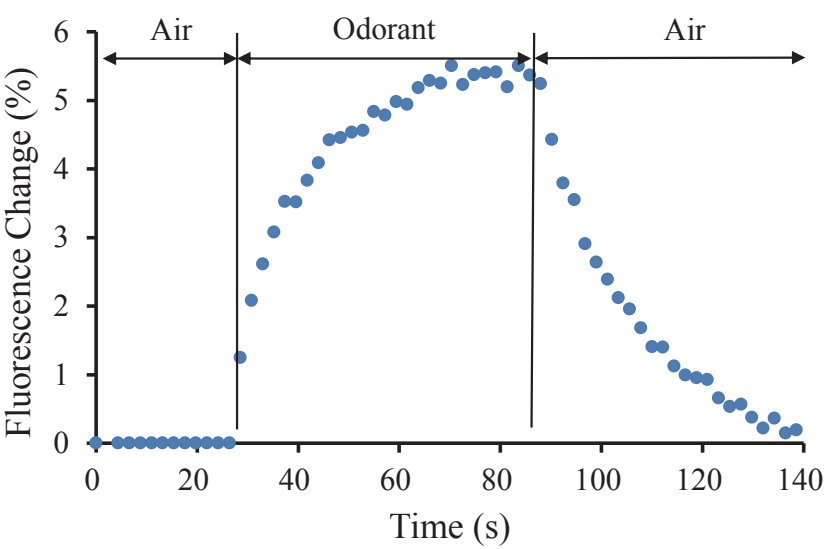

(b)

Fig. 3. (Color online) Odorant visualization based on the fluorescent micropowder film. (a) Visualization images of the film for HA and PA. (b) Time response character of the film extracted from sequential images of PA. 
molecules. In this study, the molecular imprinting effect is attributed to the strong hydrogen bonding interaction between the organic acid template and the methacrylic acid molecules. Figure 4 illustrates the GC/MS evaluation process and the results for the HA-MIP micropowder. The imprinting factor was defined by the ratio of $T I C_{\mathrm{MIP}}$ to $T I C_{\mathrm{NIP}}$. TIC is the value of total ion current obtained from the GC/MS measurement. The HA-MIP micropowder showed an IF value of 1.95 for HA, while the value for PA was only 0.68. This result confirms the molecular imprinting effect of the micropowder materials.

The selective visualization of the fluorescent MIP micropowder on odorant flow is demonstrated in Fig. 5. Although the HA-MIP micropowder film showed a response to both HA and PA flows, the fluorescence intensity change for HA was obviously higher than that for PA. The selectivity was evaluated by the comparison of the change in the ratio of the fluorescence of the MIP film to the NIP film. The MGV was extracted from the circled area in the visualized images. In the case of HA flow, the change ratio of the MIP film to the NIP film was about 1.27, while the value was only 0.47 in the case of the PA flow. The response of the NIP micropowder was attributed to the nonspecific adsorption of odorants on the micropowder surface. For the HA-MIP micropowder, more HA molecules could be absorbed into the polymer matrix due to hydrogen bonding interaction and the cavity effect, thus affording a stronger fluorescence change. PA was not the target molecule of the HA-MIP matrix. Therefore, the absorbance of the micropowder on PA was not specific and the fluorescence change was relatively poor. In addition, the recovery experiment confirmed that

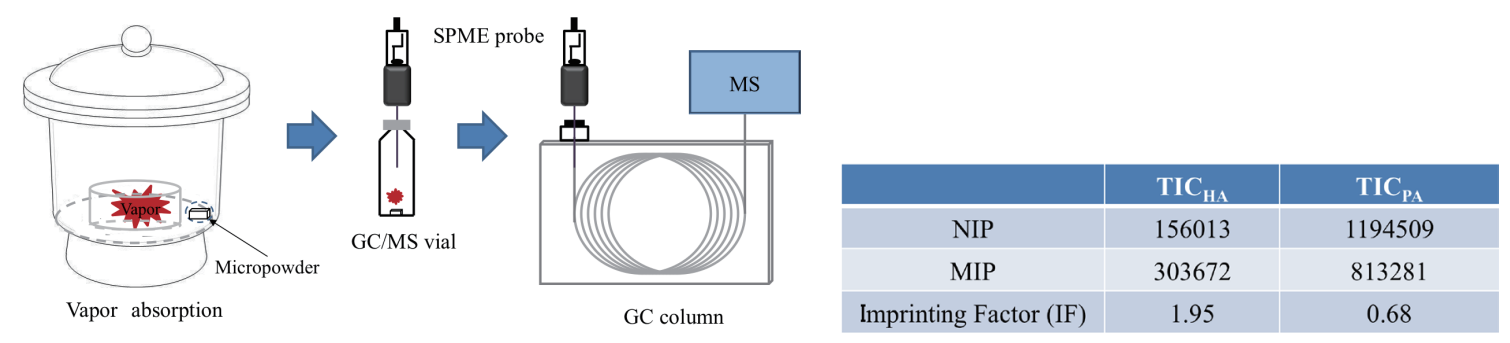

Fig. 4. (Color online) Odorant absorption character of HA-MIP micropowder evaluated by SPME-GC/MS. TIC is the total ion current obtained from the GC/MS measurement. IF is defined by the ratio of $T I C_{\mathrm{MIP}}$ to $T I C_{\mathrm{NIP}}$.

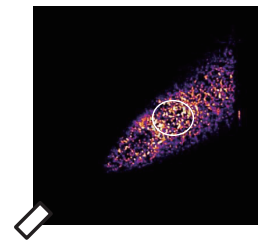

NIP

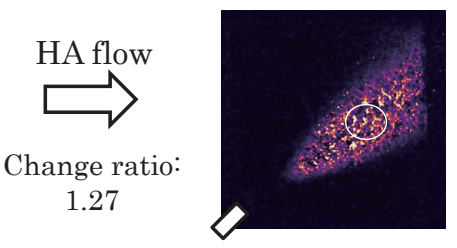

HA-MIP

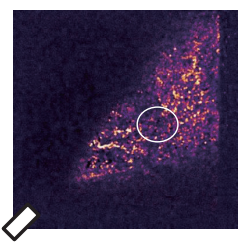

NIP

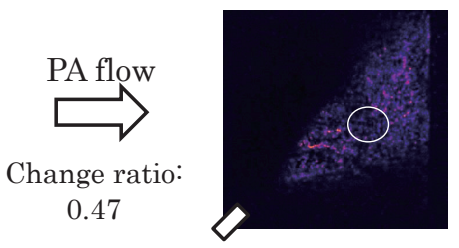

HA-MIP

(a)

(b)

Fig. 5. (Color online) Visualized images of HA-MIP and NIP micropowders on (a) HA and (b) PA. The change ratios of fluorescence intensity $(\mathrm{MGV})$ in the circled area were compared to evaluate the selective fluorescence response. The time of odorant flow was $200 \mathrm{~s}$. 
the fluorescence change of the HA-MIP micropowder for the HA flow was reversible, which is the same as that observed in Fig. 3. Although this was just a preliminary study, the result confirmed that it is possible to realize the selective and reversible odorant visualization by the combination of fluorescence dyeing and molecular imprinting techniques. Our future work will focus on the development of fluorescence multiple MIP micropowder film arrays with much higher performance. By further increasing the MIP and fluorescence effect of the micropowder materials, visualization films with higher response and recognition ability can be expected.

\section{Conclusion}

Fluorescent MIP micropowders were prepared by bulk polymerization in the presence of template and probe molecules. The fluorescence emission character and the molecular imprinting effect of the prepared micropowders were confirmed by fluorescence microscope observations and SPME-GC/MS measurements, respectively. The dyed MIP micropowders were coated on glass substrates and used as sensing films for the selective visualization of odorant flows. The selectivity was evaluated by the change in the ratio of the fluorescence of the MIP film to the NIP film. A higher ratio was demonstrated by the MIP film on the target odorant than the non-target odorant, which verified the selective visualization ability of the prepared micropowder. Compared with the previously reported fluorescence gel film, the fluorescent-dyed micropowder film showed much greater potential in odorant visualization with selectivity and reversibility.

\section{References}

P. Batog and A. Wolczowski: Eurosensor 201247 (2012) 1442.

2 A. Gomez-Marin, B. J. Duistermars, M. A. Frye, and M. Louis: Frontiers in Cellular Neuroscience 4 (2010) 6.

3 H. Ishida, T. Nakamoto, and T. Moriizumi: Sens. Actuators, B 49 (1998) 52.

4 H. Ishida, H. Tanaka, H. Taniguchi, and T. Moriizumi: Autonomous Robots 20 (2006) 231.

5 H. Ishida, Y. Wada, and H. Matsukura: IEEE Sens. J. 12 (2012) 3163.

6 J. Li, Q. Meng, Y. Wang, and M. Zeng: Autonomous Robots 30 (2011) 281.

7 K. Song, Q. Liu, and Q. Wang: Sensors 11 (2011) 2129.

8 H. Ishida, T. Tokufumi, T. Nakamoto, and T. Moriizumi: Sens. Actuators, B 83 (2002) 256.

9 H. Ishida, T. Yamanaka, N. Kushida, T. Nakamoto, and T. Moriizumi: Sens. Actuators, B 65 (2000) 14.

10 Y. Furusawa, R. Yokoyama, C. Liu, and K. Hayashi: IEEJ Trans. Sens. Micromachines 133 (2013) 199.

11 C. Liu, R. Yokoyama, S. Uchida, K. Nakano, and K. Hayashi: Proc. IEEE Sensors 2013 (DOI: 10.1109/ ICSENS.2013.6688508).

12 C. Liu and K. Hayashi: Flavour Fragrance J. 29 (2014) 356.

13 C. Liu, Y. Furusawa, and K. Hayashi: Sens. Actuators, B 183 (2013) 117.

14 H.-T. Yoshioka, C. Liu, and K. Hayashi: Sens. Actuators, B 220 (2015) 1297. 